\title{
Cancer Detection Rates in Targeted Transperineal MRI-TRUS Elastic Fusion-guided Prostate Biopsies Performed Under Local Anesthesia
}

\author{
MACIEJ JACEWICZ ${ }^{1,2}$, ERIK RUD ${ }^{3}$, KRISTINA FLOR GALTUNG ${ }^{2,3}$, DANIYAL NOOR ${ }^{3}$ and EDUARD BACO ${ }^{1}$ \\ ${ }^{1}$ Department of Urology, Oslo University Hospital, Oslo, Norway; \\ ${ }^{2}$ University of Oslo, Oslo, Norway; \\ ${ }^{3}$ Department of Radiology, Oslo University Hospital, Oslo, Norway
}

\begin{abstract}
Background/Aim: The aim of this study was to evaluate the cancer detection rate (CDR) using magnetic resonance imaging-transrectal ultrasound (MRI-TRUS) fusion-guided transperineal targeted biopsy (TB). Patients and Methods: We included 401 consecutive patients, of which 161 were biopsy-nä̈ve. All underwent prebiopsy bi-parametric MRI; patients with positive MRI [prostate imaging reporting and data system (PI-RADS $\geq 3)]$ underwent TB. Biopsy-naïve patients with positive MRI underwent TB and systematic biopsies (SBS). MRI-negative patients underwent SBs. Clinically significant prostate cancer ( $c S P C a$ ) was defined as $I S U P \geq 2$. The added value of $S B$ was defined as an upgrade from a negative biopsy or ISUP of 1 in TB to csPCa in SB. Results: The median (interquartile range) age was 69 (range $=63-74)$ years, and PSA was $6.9($ range $=4.5-11) \mathrm{ng} / \mathrm{ml}$. The overall CDR was $65 \%$, with csPCa occurring in $48 \%$. In cases of PI-RADS 5, CDR was $91 \%$, and csPCa was $77 \%$. The added value of $S B$ was $2 \%$. Conclusion: Transperineal TB biopsies using MRI-TRUS fusion yield a high CDR.
\end{abstract}

Multiparametric magnetic resonance imaging (mpMRI) may detect and localize clinically significant (cs) prostate cancer (PCa) with high precision $(1,2)$. Targeted transrectal biopsies using different navigation systems yield high cancer

This article is freely accessible online.

Clinical trial: Norwegian Randomized Trial for Evaluating Antibiotic Prophylaxis in Prostate Biopsy (NORAPP).

Correspondence to: Maciej Jacewicz, Department of Urology, Oslo University Hospital, Trondheimsveien 235, 0586, Oslo, Norway. Tel: +47 46697909, e-mail: maciejjacewicz@gmail.com

Key Words: Prostate cancer, MRI-TRUS image fusion, cancer detection rate, transperineal prostate biopsy, outpatient setting, local anesthesia. detection rates (CDRs) but are associated with significant infections (3-5). It is well known that transperineal prostate biopsies cause significantly fewer infections (6-8), but few studies have evaluated the performance of transperineal targeted biopsies (TBs) using image fusion. The aim of this study was to evaluate the CDR using MRI-transrectal ultrasound (TRUS) fusion-guided transperineal TB.

\section{Patients and Methods}

Patients and inclusion. A total of 550 patients were prospectively included in a two-center randomized control study on the role of antibiotic prophylaxis when using transperineal TB with MRITRUS fusion. The results reported in this paper are based on 401 consecutive patients from Oslo University Hospital (OUH). The inclusion period was from November 2019 to March 2021. The study was approved by the Regional Committees for Medical and Health Research Ethics (2019/1266) and has been registered at ClinicalTrials.gov (NCT04146142). Written informed consent was obtained from all patients prior to inclusion. Biopsies were performed at the Department of Urology, OUH.

Inclusion criteria included: Elevated PSA or PSA density $>0.15$ $\mathrm{ng} / \mathrm{ml}^{2}$, pathological digital rectal exploration (DRE), patients under active surveillance (AS) scheduled for routine re-biopsy, suspicion of recurrence after external beam radiation treatment (EBRT), routine biopsy at one year after partial prostate ablation using high-intensity focused ultrasound (HIFU). Exclusion criteria included: A high risk of post-biopsy infection and an allergy to the study drug. Endpoints included: CDR of anyPCa and csPCa at TB, maximum cancer core length (MCCL), and added value of SB.

MRI. Pre-biopsy MRI of the prostate was performed in all patients. At OUS, we used a 1.5T AvantoFit MRI system (Siemens Healthineers, Erlangen, Germany) with a bi-parametric protocol including high-resolution 3DT2 weighted images with multiplanar reconstruction and axial diffusion-weighted images (DWI). In 9\% of cases, MRI was performed at external radiological centers using similar bi-parametric protocols. All examinations were performed without an endorectal coil. Interpretation was done in accordance with the Prostate Imaging Reporting and Data System (PI-RADS v2) (2). MRI was classified as positive in case of PI-RADS $\geq 3$. The 

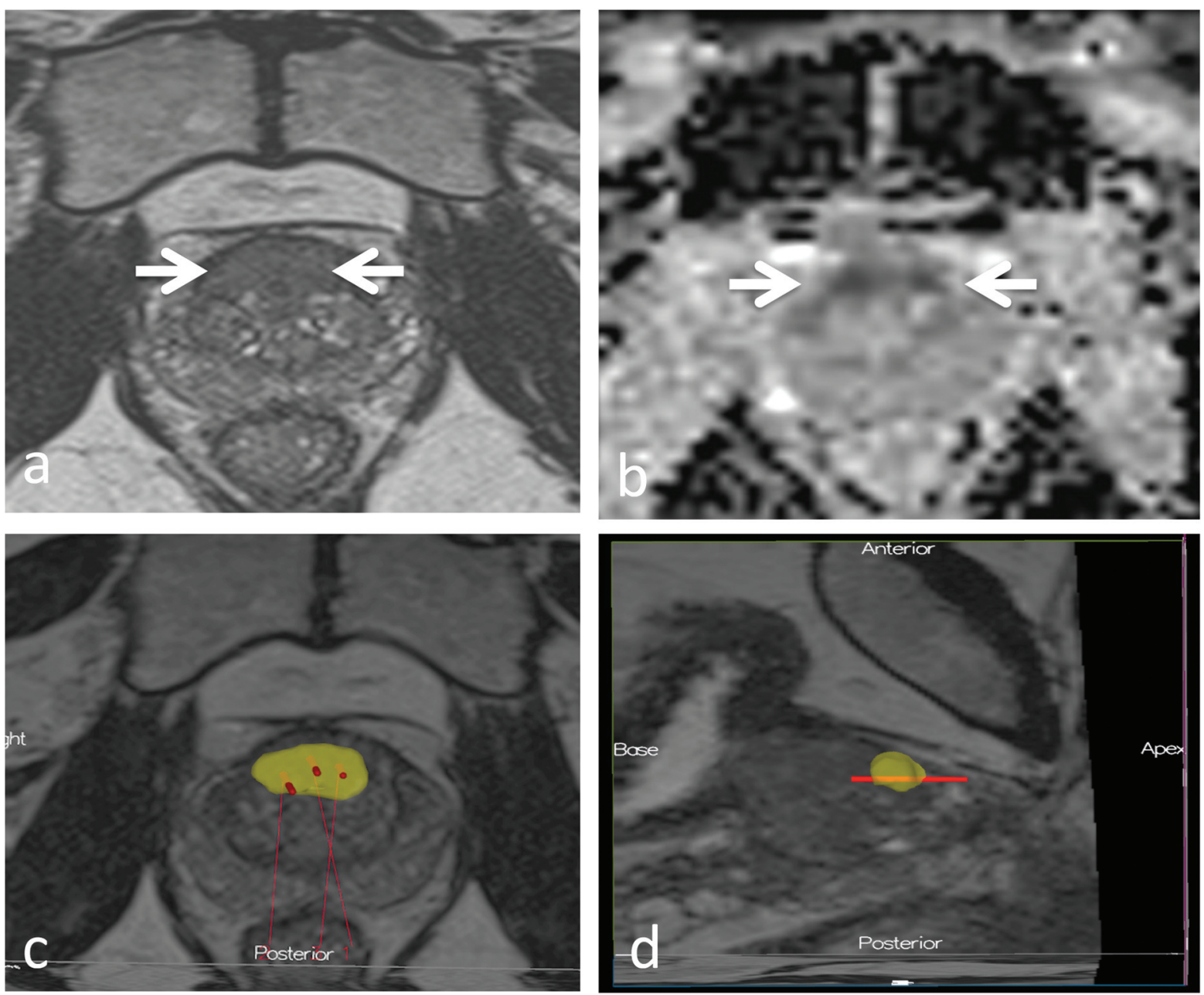

Figure 1. Magnetic resonance imaging (MRI) findings and performed target biopsies. A 58-year-old man (PSA: $11 \mathrm{ng} / \mathrm{ml}$; prostate volume: $34 \mathrm{ml})$ underwent bi-parametric MRI of the prostate, demonstrating a 30×25×15 mm PI-RADS 5 lesion (white arrows) localized anterior in the prostate's apex and middle part on T2 WI and ADC map (A, B). Patient had primarily undergone 4 cores MRI- transrectal ultrasound (TRUS) cognitive fusion guided transrectal biopsies with negative findings and 10 systematic random biopsies, of which one core revealed $2.5 \mathrm{~mm}$ prostate cancer ISUP 1. Due to the discrepancy between PI-RAD 5 suspicion in MRI and histological findings, MRI-TRUS elastic fusion and organ tracking-based transperineal targeted biopsies were performed. Target cores (red bars) oriented toward the lesion (yellow ellipse) on axial (C) and sagittal (D) MRI-TRUS fusion image diagnosed prostate cancer with ISUP 1 in 6, 6, and 7 mm, respectively. This patient was treated with MRI thermometry-guided transurethral prostate ablation.

index tumor was defined as the largest tumor in T2W images with the lowest apparent diffusion coefficient (9).

Biopsy procedure. The MRI prostate volume was segmented, and the index tumor was annotated in the $3 \mathrm{~T} 2 \mathrm{w}$ images. An elastic image fusion and organ tracking-based navigation system was used for all biopsies (Koelis ${ }^{\circledR}$ Trinity Perine, Meylan, France), using a free-hand technique with a linear guide fixated to the TRUS transducer (Figure 1).

Patients were placed on a surgical table with legs placed in stirrups to obtain a lithotomy position. The scrotum was elevated and fixated with a sterile drape (Steri-Draps ${ }^{\mathrm{TM}}$ ). Shaving of the perineum was performed where necessary. Pre-biopsy surgical disinfection was performed using cotton swabs soaked in chlorhexidine (Fresenius Kabi, Oslo, Norway).

Local anesthesia (LA) was achieved using $1 \%$ lidocaine blended with $4 \mathrm{ml}$ of $8.4 \%$ sodium bicarbonate. $\mathrm{NaHCO}_{3}$ was used to achieve a physiological $\mathrm{pH}$ of 7 , in order to reduce discomfort. Twenty $\mathrm{ml}$ of the solution was placed cutaneously/subcutaneously in a fan shape $2 \mathrm{~cm}$ above the anus. Deep anesthesia was achieved using $20 \mathrm{ml}$ of solution placed at the prostatic apex, bilaterally in the levator ani, and along the path of the needle. An automated 3D side-fire ultrasound probe was introduced into the rectum (Koelis Steady Pro, France) allowing full freedom of movement.

A minimum of one biopsy core TB was obtained from the index tumor. In cases of biopsy-naïve patients or those with negative MRI, 


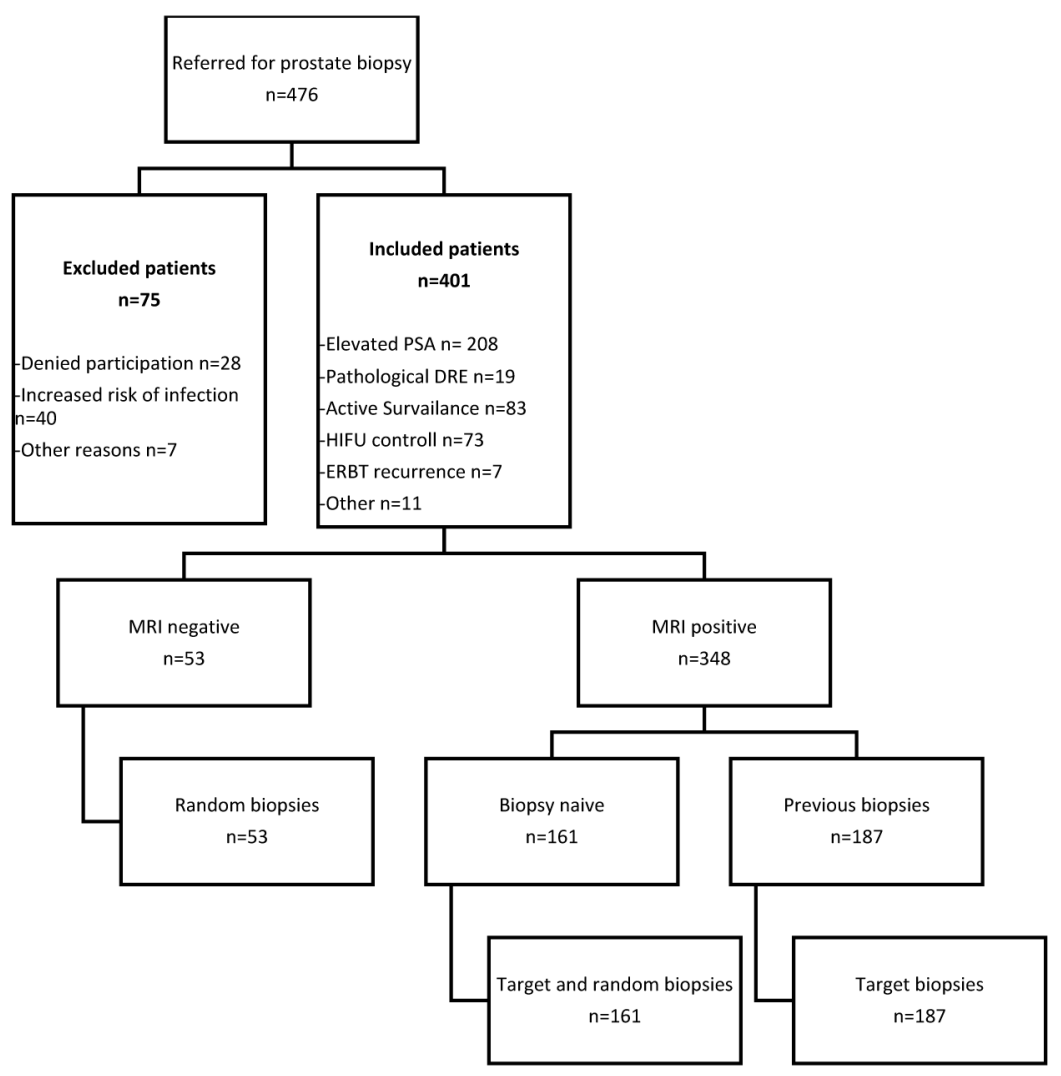

Figure 2. Flowchart of included and excluded patients.

6-12 additional systematic biopsies (SBs) were performed. 3D registration of each biopsy trajectory allowed for adequate distribution and registration of all cores, providing a 3D digitalized biopsy cartography.

One hour was set aside for preparation, consultation, and biopsy procedure, probe in/out time was estimated to 20-30 min. Biopsy time itself was about 5-10 min.

The procedure was performed by two urologists with 11 and 6 years of experience performing TBs using image fusion, as well as one urological resident who was a novice to the technique at the start of inclusion.

Histology. The number of biopsies and location of each biopsy core was noted on the pathology form in accordance with the 3D registration from the Koelis Trinity device. Results are reported in accordance with the International Society of Uropathology (ISUP grade group), from ISUP grade group (GG) 1-5 (10). Clinically significant prostate cancer (csPCa) was defined as $\mathrm{GG} \geq 2$. An added value of $\mathrm{SB}$ was defined as an upgrade from a negative or GG 1 at $\mathrm{TB}$ to csPCa in SB.

Statistics. Patient characteristics were described using median values with interquartile ranges (IQRs). Cancer detection rates with $95 \%$ confidence intervals are reported. The maximum cancer core lengths are reported as median values with IQRs. All analyses were performed using IBM SPSS v27 (IBM, Armonk, NY, USA) and MedCalc version 16.2 (MedCalc Software, Ostend, Belgium).

\section{Results}

The results from 401 patients were included in the analysis, of which $40 \%$ (161/401) were biopsy-naïve (Figure 2). The median $(I Q R)$ age was 69 (range=63-74) years with a median PSA of 6.9 (4.5-11) ng/ml. The overall median PSA densities were $0.17 \mathrm{ng} / \mathrm{ml}^{2}$ and $0.24 \mathrm{ng} / \mathrm{ml}^{2}$ in cases of PI-RADS 5 . Clinical data are specified in accordance with the PI-RADS score of the index tumor in Table I. The median time from MRI to biopsy was 27 days (range=10-59).

MRI was positive in $87 \%$ of patients (348/401). Index tumor was located in the peripheral zone in $80 \%$ (278/348) and in the transitional zone in $20 \%$ of cases (70/348) (Table II). TBs were performed in all MRI-positive patients, and 46\% (161/348) underwent additional SBs. The median (IQR) number of TB and SB was 4 (range=3-4) and 5 (range=3-7), respectively. In MRI-negative patients, the number of SBs was 10 (10-12). The index tumors' median (IQR) biopsy core length was 14 (range $=11-15) \mathrm{mm}$, and MCCL was 8 (range=4-10) $\mathrm{mm}$. In 
Table I. Clinical data in accordance to PIRADS score.

\begin{tabular}{|c|c|c|c|c|c|c|c|c|c|c|}
\hline \multicolumn{3}{|c|}{ PI-RADS index tumor } & \multicolumn{2}{|c|}{ Prostate volume $(\mathrm{ml})$} & \multicolumn{2}{|c|}{ Tumor volume (ml) } & \multicolumn{2}{|c|}{ PSA (ng/ml) } & \multicolumn{2}{|c|}{ PSA density } \\
\hline & $\mathrm{n}$ & $\%$ & Median & IQR & Median & IQR & Median & IQR & Median & IQR \\
\hline $1-2$ & 53 & 13 & 26 & $16-36$ & n.a & n.a & 2.9 & $1.2-6.8$ & 0.11 & $0.05-0.21$ \\
\hline 3 & 74 & 19 & 49 & $32-70$ & 0.3 & $0.13-0.50$ & 6.2 & 4.4-9.1 & 0.12 & $0.08-0.22$ \\
\hline 4 & 137 & 34 & 44 & $31-62$ & 0.5 & $0.26-0.85$ & 6.7 & $4.5-10$ & 0.16 & $0.1-0.24$ \\
\hline 5 & 137 & 34 & 38 & $29-50$ & 2.0 & $1.02-4.42$ & 9.6 & $5.9-14$ & 0.24 & $0.15-0.38$ \\
\hline Total & 401 & 100 & 40 & $27-58$ & 0.7 & $0.32-1.99$ & 6.9 & $4.5-11$ & 0.17 & $0.10-0.28$ \\
\hline
\end{tabular}

PSA: Prostate specific antigen; IQR: interquartile range; PI-RADS: prostate imaging reporting and data system.

SBs, the biopsy core length was 12 (range=10-15) $\mathrm{mm}$, and MCCL was 4 (range=2-6) $\mathrm{mm}$. The overall CDR of anyPCa was $65 \%(260 / 401,95 \% \mathrm{CI}=57-73)$, and CDR for csPCa was $48 \%(194 / 401,95 \% \mathrm{CI}=42-56)$. In the index tumor, anyPCa was found in $66 \%(231 / 348,95 \% \mathrm{CI}=58-76)$, and csPCa was found in $53 \%(183 / 348,95 \% \mathrm{CI}=45-61)$. In PI-RADS 5 lesions, anyPCa was found in $91 \%(125 / 137,95 \% \mathrm{CI}=76-100)$, and csPCa was found in 77\% $(106 / 137,95 \% \mathrm{CI}=63-95)$. Table III demonstrates the CDR according to PI-RADS score.

The added value of SB was $2 \%(3 / 161,95 \% \mathrm{CI}=0-5)$. In $1 \%$ of cases $(1 / 161,95 \% \mathrm{CI}=0-3)$, the $\mathrm{TB}$ was negative, while SB detected csPCa. In $1 \%$ of cases, TB showed a GG 1, while SB demonstrated GG 2. A higher GG was detected in the SB in $2 \%(4 / 161,95 \% \mathrm{CI}=1-6)$ of those with csPCa in TB. In MRI-negative patients, the CDRs for anyPCa and csPCa were $30 \%(16 / 53,95 \% \mathrm{CI}=17-49)$ and $17 \%(9 / 53$, $95 \% \mathrm{CI}=8-32$ ), respectively. All cases of csPCa were found in patients previously treated with HIFU. In 8\% (4/53), csPCa was found in the treated lobe.

\section{Discussion}

In this study, the overall CDR of anyPCa was $65 \%$ with $91 \%$ in PI-RADS 5 tumors when using transperineal TBs and image fusion under local anesthesia. These results are consistent with our previous multicenter study, which demonstrated 94\% anyPCa in PI-RADS 5 tumors when using the same technique (8).

A recent systematic review by Bhavan et al. reported that transperineal technique achieved higher CDRs than transrectal biopsies, and had lower infection rates (11). Therefore, a transperineal technique should be preferred over a transrectal approach.

In our study, the added value of SB in addition to TB for detecting csPCa was $2 \%$. For comparison, Exterkate et al. reported an added value of $1.3 \%$ in SB in patients with a prior negative SB (12). In contrast, other studies report 4-5\% added value of $\mathrm{SB}$ as an addition to $\mathrm{TB}$ in biopsy-naïve
Table II. Biopsy results according to location.

\begin{tabular}{lcccc}
\hline \multicolumn{5}{c}{ Index tumor } \\
\hline & $\mathrm{n}$ & CDR & $\mathrm{n}$ & $\%$ \\
\hline \multirow{2}{*}{ Peripheral zone } & \multirow{2}{*}{278} & Any PCa & 179 & 64 \\
& & csPCa & 135 & 49 \\
Tranzitional zone & \multirow{2}{*}{70} & Negative & 99 & 36 \\
& & Any PCa & 52 & 74 \\
& & csPca & 39 & 56 \\
& & Negative & 18 & 26 \\
\hline
\end{tabular}

Pca: Prosate cancer; csPCA: clinically significant prostate cancer; CDR: cancer detection rate.

patients $(13,14)$. The reasons for these variations are unknown but may be due to patient selection and different numbers of SB, which were lower in our study than in the study by van der Leest et al. (5 vs. 12) (13). We also used image fusion in all patients, while the MRI-FIRST study used image fusion in only $30 \%$ (14). By not using image fusion, it is possible that the $\mathrm{SB}$ detected csPCa that was accidentally missed by the TB. The European Association of Urology (EAU) currently recommends SB as an addition to TB in biopsy-naïve patients (15).

A recent randomized trial demonstrated non-inferiority of TB compared to SB in biopsy naïve men for detecting csPCa (16). Although SB may not surely improve CDRs, they may provide better risk stratification and patient selection for appropriate treatments such as focal therapy (17). SB may also increase the number of positive cores, which is relevant in risk-classification systems such as the National Comprehensive Cancer Network (18).

In our study, SBs were performed in all 53 MRI-negative patients, and csPCa was found in $17 \%$. This is higher than that in other studies reporting on biopsy-naïve patients. According to a Cochrane meta-analysis, SBs detect csPCa in $8 \%$ of biopsy-naïve patients with a negative MRI result (19). 
Table III. Biopsy results in accordance to PI-RADS score in index tumor.

\begin{tabular}{|c|c|c|c|c|c|c|c|c|c|c|c|c|c|c|c|c|c|}
\hline \multirow[t]{3}{*}{ PI-RADS } & \multirow[t]{3}{*}{ Total } & \multicolumn{16}{|c|}{ ISUP Grade Group Index Tumor } \\
\hline & & \multicolumn{2}{|c|}{ Negative } & \multicolumn{2}{|c|}{1} & \multicolumn{2}{|c|}{2} & \multicolumn{2}{|c|}{3} & \multicolumn{2}{|c|}{4} & \multicolumn{2}{|c|}{5} & \multicolumn{2}{|c|}{ Any PCa } & \multicolumn{2}{|c|}{$\mathrm{csPCa}$} \\
\hline & & $\mathrm{n}$ & $\%$ & $\mathrm{n}$ & $\%$ & $\mathrm{n}$ & $\%$ & $\mathrm{n}$ & $\%$ & $\mathrm{n}$ & $\%$ & $\mathrm{n}$ & $\%$ & $\mathrm{n}$ & $\%$ & $\mathrm{n}$ & $\%$ \\
\hline $1-2$ & 53 & 37 & 70 & 6 & 11 & 5 & 9 & 2 & 4 & 2 & 4 & 0 & 0 & 16 & 30 & 9 & 17 \\
\hline 3 & 74 & 51 & 69 & 8 & 11 & 12 & 16 & 2 & 3 & 1 & 1 & 0 & 0 & 23 & 31 & 15 & 20 \\
\hline 4 & 137 & 54 & 39 & 30 & 22 & 27 & 20 & 10 & 7 & 11 & 8 & 5 & 4 & 83 & 61 & 53 & 39 \\
\hline 5 & 137 & 12 & 9 & 19 & 14 & 43 & 31 & 24 & 18 & 17 & 12 & 22 & 16 & 125 & 91 & 106 & 77 \\
\hline Total & 401 & 117 & & 57 & & 82 & & 36 & & 29 & & 27 & & 231 & 66 & 183 & 46 \\
\hline
\end{tabular}

Pca: Prostate cancer; csPCa: clinically significant prostate cancer; ISUP: International Society of Urological Pathology; PI-RADS: prostate imaging reporting and data system.

However, in our study, all cases of cancer were found in MRI-negative patients who had previously been treated for csPC using HIFU. Since our MRI-negative group was heterogeneous and highly selected, comparison is difficult.

In our study, the median MCCL of TB and SB was $8 \mathrm{~mm}$ and $4 \mathrm{~mm}$, respectively. The median MCCL in patients with a negative MRI was $3 \mathrm{~mm}$, indicating that SB detect smallvolume cancers of uncertain clinical importance. Other studies report similar results, where the MCCL of target biopsies was a significant predictor of cancer volume and pathological Tstage, while no such correlation was demonstrated for SB (20, $21)$. Studies also report that SBs reveal smaller cancer volumes of uncertain clinical benefit, and most report that SB increase the risk of detecting insignificant cancer $(16,22)$.

Limitations. This study was limited in that the population was heterogeneous and included biopsy-naïve patients, patients under active surveillance, and patients previously treated for $\mathrm{PCa}$. This makes it difficult to compare CDRs and the added value of SB to studies reporting on biopsy-naïve or re-biopsy patients only. Furthermore, we used a bi-parametric MRI protocol $(\mathrm{T} 2 \mathrm{w}+\mathrm{DWI})$, while the recommended standard is multiparametric MRI (T2w+DWI+DCE). This may have affected the overall CDR in this study. The biopsy strategy was not standardized for all patients, and the numbers of TBs and SBs performed were based on clinical judgment. Although this may have affected the CDR in this study, it represents clinical practice.

\section{Conclusion}

Transperineal prostate biopsy using a free-hand MRI-TRUS fusion technique under local anesthesia in an outpatient setting is a highly accurate diagnostic tool in prostate cancer diagnostics. Transperineal TB offer equally high detection rates compared to transrectal $\mathrm{TB}$; because of significantly less infections, a transperineal approach should be preferred.

\section{Conflicts of Interest}

The Authors declare no conflicts of interest in relation to this study.

\section{Authors' Contributions}

Maciej Jacewicz: Conceptualization, methodology, formal analysis, investigation, data curation, writing, project administration. Eduard Baco: Conceptualization, methodology, investigation, writing, supervision. Erik Rud: Conceptualization, formal analysis, data curation, writing. Daniyal Noor, Kristina Flor Galtung: Writing review \& editing.

\section{References}

1 van der Leest M, Israël B, Cornel EB, Zámecnik P, Schoots IG, van der Lelij H, Padhani AR, Rovers M, van Oort I, Sedelaar M, Hulsbergen-van de Kaa C, Hannink G, Veltman J and Barentsz $\mathrm{J}$ : High diagnostic performance of short magnetic resonance imaging protocols for prostate cancer detection in biopsy-naïve men: The next step in magnetic resonance imaging accessibility. Eur Urol 76(5): 574-581, 2019. PMID: 31167748. DOI: 10.1016/j.eururo.2019.05.029

2 Greer MD, Brown AM, Shih JH, Summers RM, Marko J, Law YM, Sankineni S, George AK, Merino MJ, Pinto PA, Choyke PL and Turkbey B: Accuracy and agreement of PIRADSv2 for prostate cancer mpMRI: A multireader study. J Magn Reson Imaging 45(2): 579-585, 2017. PMID: 27391860. DOI: 10.1002/ jmri.25372

3 Baco E, Rud E, Eri LM, Moen G, Vlatkovic L, Svindland A, Eggesb $\varnothing \mathrm{HB}$ and Ukimura O: A randomized controlled trial to assess and compare the outcomes of two-core prostate biopsy guided by fused magnetic resonance and transrectal ultrasound images and traditional 12-core systematic biopsy. Eur Urol 69(1): 149-156, 2016. PMID: 25862143. DOI: 10.1016/j.eururo. 2015.03.041

4 Grummet JP, Weerakoon M, Huang S, Lawrentschuk N, Frydenberg M, Moon DA, O'Reilly M and Murphy D: Sepsis and 'superbugs': should we favour the transperineal over the transrectal approach for prostate biopsy? BJU Int 114(3): 384388, 2014. PMID: 24612341. DOI: 10.1111/bju.12536 
5 Williamson DA, Roberts SA, Paterson DL, Sidjabat H, Silvey A, Masters J, Rice M and Freeman JT: Escherichia coli bloodstream infection after transrectal ultrasound-guided prostate biopsy: implications of fluoroquinolone-resistant sequence type 131 as a major causative pathogen. Clin Infect Dis 54(10): 14061412, 2012. PMID: 22419681. DOI: $10.1093 / \mathrm{cid} / \mathrm{cis} 194$

6 Stefanova V, Buckley R, Flax S, Spevack L, Hajek D, Tunis A, Lai E, Loblaw A and Collaborators: Transperineal prostate biopsies using local anesthesia: Experience with 1,287 patients. Prostate Cancer Detection Rate, Complications and Patient Tolerability. J Urol 201(6): 1121-1126, 2019. PMID: 30835607. DOI: 10.1097/JU.0000000000000156

7 Bhanji Y, Allaway MJ and Gorin MA: Recent advances and current role of transperineal prostate biopsy. Urol Clin North Am 48(1): 25-33, 2021. PMID: 33218591. DOI: 10.1016/j.ucl. 2020.09.010

8 Jacewicz M, Günzel K, Rud E, Lauritzen PM, Galtung KF, Hinz S, Magheli A and Baco E: Multicenter transperineal MRI-TRUS fusion guided outpatient clinic prostate biopsies under local anesthesia. Urol Oncol 39(7): 432.e1-432.e7, 2021. PMID: 33257219. DOI: 10.1016/j.urolonc.2020.11.009

9 Rud E, Klotz D, Rennesund K, Baco E, Berge V, Lien D, Svindland A, Lundeby E, Berg RE, Eri LM and Eggesbø HB: Detection of the index tumour and tumour volume in prostate cancer using T2-weighted and diffusion-weighted magnetic resonance imaging (MRI) alone. BJU Int 114(6b): E32-E42, 2014. PMID: 24447606. DOI: 10.1111/bju.12637

10 Egevad L, Delahunt B, Srigley JR and Samaratunga H: International Society of Urological Pathology (ISUP) grading of prostate cancer - An ISUP consensus on contemporary grading. APMIS 124(6): 433435, 2016. PMID: 27150257. DOI: 10.1111/apm.12533

11 Rai BP, Mayerhofer C, Somani BK, Kallidonis P, Nagele U and Tokas T: Magnetic resonance imaging/ultrasound fusion-guided transperineal versus magnetic resonance imaging/ultrasound fusionguided transrectal prostate biopsy-a systematic review. Eur Urol Oncol, 2021. PMID: 33478936. DOI: 10.1016/j.euo.2020.12.012

12 Exterkate L, Wegelin O, Barentsz JO, van der Leest MG, Kummer JA, Vreuls W, de Bruin PC, Bosch JLHR, van Melick HHE and Somford DM: Is there still a need for repeated systematic biopsies in patients with previous negative biopsies in the era of magnetic resonance imaging-targeted biopsies of the prostate? Eur Urol Oncol 3(2): 216-223, 2020. PMID: 31239236. DOI: 10.1016/j.euo.2019.06.005

13 van der Leest M, Cornel E, Israël B, Hendriks R, Padhani AR, Hoogenboom M, Zamecnik P, Bakker D, Setiasti AY, Veltman J, van den Hout $\mathrm{H}$, van der Lelij $\mathrm{H}$, van Oort I, Klaver S, Debruyne F, Sedelaar M, Hannink G, Rovers M, Hulsbergen-van de Kaa C and Barentsz JO: Head-to-head comparison of transrectal ultrasound-guided prostate biopsy versus multiparametric prostate resonance imaging with subsequent magnetic resonance-guided biopsy in biopsy-naïve men with elevated prostate-specific antigen: a large prospective multicenter clinical study. Eur Urol 75(4): 570-578, 2019. PMID: 30477981. DOI: 10.1016/j.eururo.2018.11.023

14 Rouvière O, Puech P, Renard-Penna R, Claudon M, Roy C, Mège-Lechevallier F, Decaussin-Petrucci M, DubreuilChambardel M, Magaud L, Remontet L, Ruffion A, Colombel M, Crouzet S, Schott AM, Lemaitre L, Rabilloud M, Grenier N and MRI-FIRST Investigators: Use of prostate systematic and targeted biopsy on the basis of multiparametric MRI in biopsy- naive patients (MRI-FIRST): a prospective, multicentre, paired diagnostic study. Lancet Oncol 20(1): 100-109, 2019. PMID: 30470502. DOI: 10.1016/S1470-2045(18)30569-2

15 Mottet N, Cornford P, van den Bergh RCN, Briers E, Expert Patient Advocate (European Prostate Cancer Coalition/Europa UOMO), De Santis M, Gillessen S, Grummet J, Henry AM, van der Kwast TH, Lam TB, Mason MD, O‘Hanlon S, Oprea-Lager DE, Ploussard G, van der Poel HG, Rouvière O, Schoots IG, Tilki D and Wiegel T: EAU Prostate Cancer Guidelines, 2020. Available at: https://uroweb.org/guideline/prostate-cancer/ [Last accessed on July 16, 2021]

16 Klotz L, Chin J, Black PC, Finelli A, Anidjar M, Bladou F, Mercado A, Levental M, Ghai S, Chang SD, Milot L, Patel C, Kassam Z, Moore C, Kasivisvanathan V, Loblaw A, Kebabdjian M, Earle CC, Pond GR and Haider MA: Comparison of multiparametric magnetic resonance imaging-targeted biopsy with systematic transrectal ultrasonography biopsy for biopsynaive men at risk for prostate cancer: a Phase 3 randomized clinical trial. JAMA Oncol 7(4): 534-542, 2021. PMID: 33538782. DOI: $10.1001 /$ jamaoncol.2020.7589

17 Ploussard G, Beauval JB, Lesourd M, Almeras C, Assoun J, Aziza R, Gautier JR, Loison G, Portalez D, Salin A, Tollon C, Soulié M, Malavaud B and Roumiguié M: Added value of concomitant systematic and fusion targeted biopsies for grade group prediction based on radical prostatectomy final pathology on positive magnetic resonance imaging. J Urol 202(6): 1182-1187, 2019. PMID: 31246548. DOI: 10.1097/JU.0000000000000418

$18 \mathrm{Xu} \mathrm{H}$, Zhu Y, Dai B and Ye DW: National Comprehensive Cancer Network (NCCN) risk classification in predicting biochemical recurrence after radical prostatectomy: a retrospective cohort study in Chinese prostate cancer patients. Asian J Androl 20(6): 551-554, 2018. PMID: 30027928. DOI: 10.4103/aja.aja_52_18

19 Drost FH, Osses DF, Nieboer D, Steyerberg EW, Bangma CH, Roobol MJ and Schoots IG: Prostate MRI, with or without MRItargeted biopsy, and systematic biopsy for detecting prostate cancer. Cochrane Database Syst Rev 4: CD012663, 2019. PMID: 31022301. DOI: 10.1002/14651858.CD012663.pub2

20 Simopoulos DN, Sisk AE Jr, Priester A, Felker ER, Kwan L, Delfin MK, Reiter RE and Marks LS: Cancer core length from targeted biopsy: an index of prostate cancer volume and pathological stage. BJU Int 124(2): 275-281, 2019. PMID: 30694605. DOI: 10.1111/bju.14691

21 Matsugasumi T, Baco E, Palmer S, Aron M, Sato Y, Fukuda N, Süer E, Bernhard JC, Nakagawa H, Azhar RA, Gill IS and Ukimura O: Prostate cancer volume estimation by combining magnetic resonance imaging and targeted biopsy proven cancer core length: correlation with cancer volume. J Urol 194(4): 957965, 2015. PMID: 25912496. DOI: 10.1016/j.juro.2015.04.075

22 Goldberg H, Ahmad AE, Chandrasekar T, Klotz L, Emberton M, Haider MA, Taneja SS, Arora K, Fleshner N, Finelli A, Perlis N, Tyson MD, Klaassen Z and Wallis CJD: Comparison of magnetic resonance imaging and transrectal ultrasound informed prostate biopsy for prostate cancer diagnosis in biopsy naïve men: a systematic review and meta-analysis. J Urol 203(6): 1085-1093, 2020. PMID: 31609177. DOI: 10.1097/JU.0000000000000595

Received June 30, 2021

Revised July 15, 2021

Accepted July 16, 2021 